\title{
Safety and efficacy of reconstituted atropine $0.01 \%$ eye drops for Indian children with myopic progression
}

\author{
Vidhya C., Kaushik Murali, Sowmya R. \\ Pediatric Ophthalmology and Strabismus Department, Sankara Eye Hospitals, \\ Bangalore, India
}

\begin{abstract}
Aim: To evaluate safety and efficacy of reconstituted atropine $0.01 \%$ eye drops for Indian children with myopic progression.

Methods: Fifty children with progressive myopia with their spherical equivalent increasing at least $0.75 \mathrm{D}$ in 6 months $(0.75$ to $1.50 \mathrm{D})$ were put on reconstituted atropine $0.01 \%$. Ocular examination, including near vision, near point of accommodation (NPA), pupil size and axial length, was done. Subjective symptoms of glare and photophobia were noted. Systemic side effects were documented. Analysis was done using Microsoft Excel 2010.

Results: The average age of patients was 9.5 years (range 5 to 14 years) and they were followed up for 1 year. Average mesopic and photopic pupil size was 5 and $4 \mathrm{~mm}$, respectively. Average NPA was $9 \mathrm{~cm}$. Mean increase in spherical equivalence was $0.18 \mathrm{D}$ over 6 months. Average increase in axial length was $0.12 \pm 0.11 \mathrm{~mm}$ over 6 months and $0.2 \pm$ $0.29 \mathrm{~mm}$ over 1 year. Average increase in spherical equivalent over 6 months was $0.07 \pm$ $0.19 \mathrm{D}$ and over 1 year was $0.32 \pm 0.29 \mathrm{D}$. No systemic side effects were recorded.

Conclusion: Reconstituted atropine $0.01 \%$ eye drops is safe and efficacious in slowing the progression of myopia in Indian children.
\end{abstract}

\section{Introduction}

Myopia is one of the most common ocular disorders in the world and considered to be the leading cause of visual impairment. ${ }^{1}$ Various prevalence studies have shown a substantial increase in myopia among adolescents and it is now thought to be approaching $10 \%$ to $25 \%$ in the West and $60 \%$ to $80 \%$ in the East. ${ }^{2}$ Various surveys in India have found myopia prevalence ranging from $6.9 \%$ to $19.7 \%$. $^{3,4}$

At present, the mechanisms involved in the aetiology of myopia are unclear and both environmental and genetic factors have been associated with the onset and progression of myopia. ${ }^{1,5,6}$

Correspondence: Dr. Vidhya C., MS, FPOS, FAICO, Pediatric Ophthalmology and Strabismus Department, Sankara Eye Hospitals, Varthur Main Road, Kundalahalli Gate, 560037 Bangalore, India.

E-mail:dr.optho.vidhya@gmail.com 
Safety and efficacy of reconstituted atropine $0.01 \%$ eye drops for Indian children with myopic progression

Atropine eye drops were first proposed as a treatment of myopia in the 1920 s. Since then, there have been numerous studies on this subject. However, evidence from randomized controlled trials has become available only over the last two decades. These trials confirm that atropine eye drops are effective in the control of myopia in a dose-dependent manner. ${ }^{7-10}$

The broad-band muscarinic antagonist, atropine, is believed to have a therapeutic effect in reducing the progression of axial myopia in humans via a non-accommodative mechanism. It is still unclear, however, whether long-term atropine usage would alter the retinal function via two possible mechanisms, namely retinal toxicity and photic retinopathy. ${ }^{11}$

While ATOM2 study showed the efficacy of low-dose atropine of $0.01 \%$, there is little evidence of low-dose atropine in preventing myopic progression in Indian children. Also, with not many commercial preparations, reconstituted atropine $0.01 \%$ as an alternative need to be studied. A study was undertaken to evaluate the safety and efficacy of reconstituted atropine $0.01 \%$ used daily in children with progressive myopia.

\section{Materials and methods}

The study was approved by our hospital's ethics committee and informed consent was obtained from the parents regarding the off-label use of the drug and that a reconstituted preparation was being used.

Reconstitution was done under aseptic precautions and laminar flow by mixing $0.1 \mathrm{ml}$ of atropine 1\% (Atrosulph Eye Drops, Entod Pharmaceuticals Ltd., Mumbai, India) into $10 \mathrm{ml}$ of carboxymethylcellulose eye drops $5 \mathrm{mg} / \mathrm{ml}$ (Extralube Eye Drops, Microlabs Limited, Mumbai, India) and the drops were to be used within 1 month of reconstitution.

A prospective study was conducted enrolling 50 children presenting to the paediatric ophthalmology outpatient department, between 5 and 15 years with a minimum base line refractive error $-2 D$ (spherical power) with progressive myopia where progression was defined as documented increase of myopia by $0.75 \mathrm{D}$ or more within a period of 6 months.

Children with severe congenital or developmental delay or systemic diseases, inability to communicate or undertake a complete ophthalmic examination or with conditions in the eye affecting visual acuity (apart from the refractive error) were excluded.

Enrolled patients underwent a comprehensive eye examination and the following tests:

- visual acuity with logMAR chart

- photopic pupil size using Optikon scout 
- near point of accommodation (NPA) using RAF (Royal Air Force) ruler

- axial length recorded using Lenstar Biometry

- macular thickness measured by spectral domain optical coherence tomography

All these values were taken as baseline values. Refractive error was documented as spherical equivalent.

All the selected children were given reconstituted atropine $0.01 \%$ eye drops to be applied one drop to each eye at night.

The above tests were repeated during each review visits every 3 months and subjective symptoms of glare to light and photophobia were elicited from the children. Findings at 6 months and at final follow-up ( 9 to 16 months) was taken for analysis.

Children and parents were also asked if the atropine eye drops were acceptable to the child as a long-term medication and if it interfered with their daily tasks resulting in discontinuation.

Any systemic side effects were elicited and documented. Possibility of contamination was ruled out by sending 10 eye drop samples for culture on blood agar when the eye drop bottle was returned after use for 1 month.

\section{Progression of spherical equivalent before and after Atropine therapy}

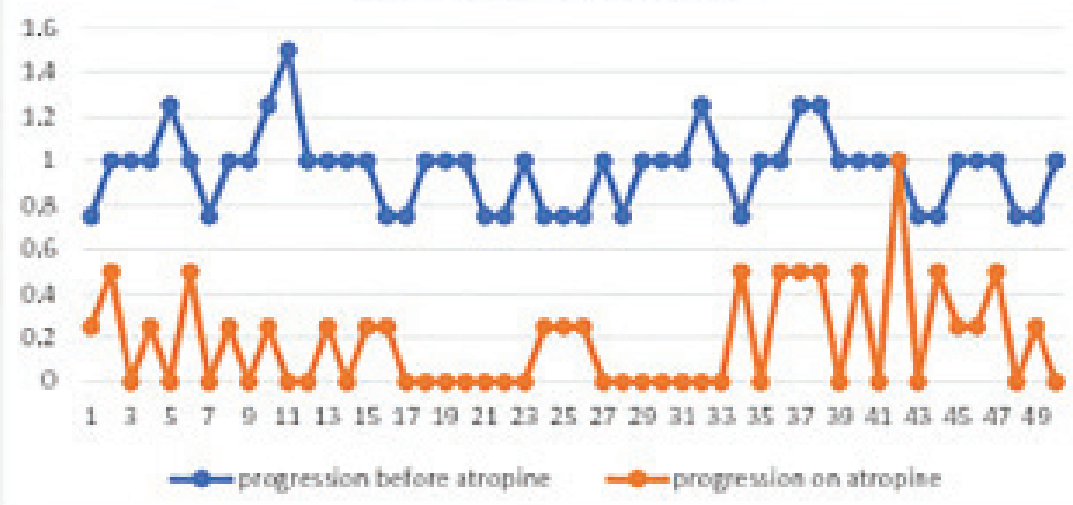

Fig. 1. Progression of spherical equivalent before and after starting atropine therapy over 6 months. 
Safety and efficacy of reconstituted atropine $0.01 \%$ eye drops for Indian children with myopic progression

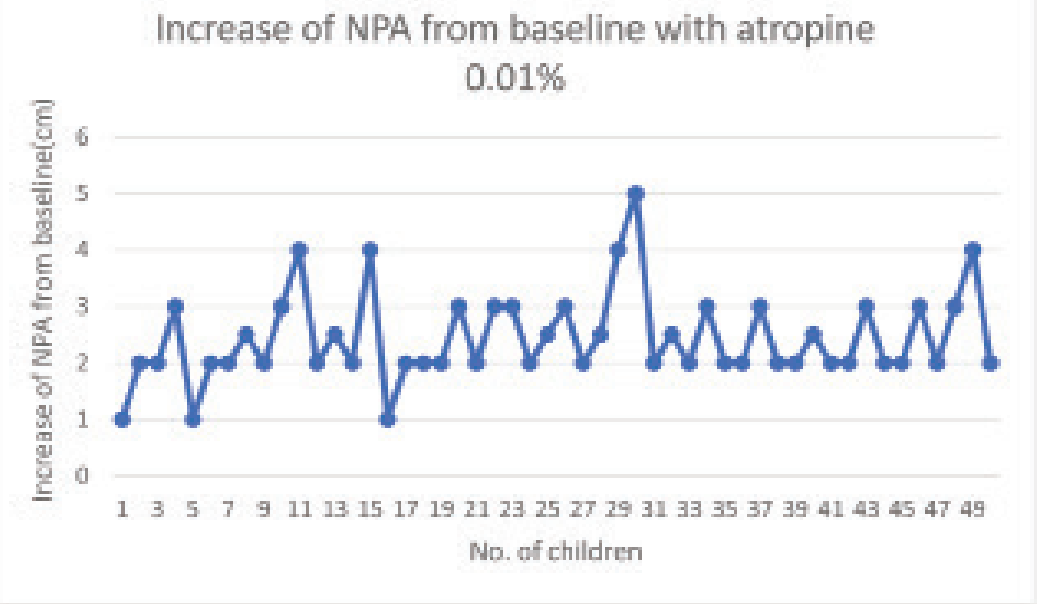

Fig. 2. Average increase of NPA $(\mathrm{cm})$ with atropine $0.01 \%$ at the end of 6 months.

\section{Results}

The average age of the patients was 9.5 years (range 5 to 14 years). The average follow-up was 1 year (range 10 to 16 months). Thirty-five children completed the 1-year follow-up.

Average spherical power was $5.5 \mathrm{D}$ (range 3 to $-9.5 \mathrm{D}$ ) with a mean progression of $1.0 \mathrm{D}$ per 6 months (range 0.75 to $1.50 \mathrm{D}$ ) before starting atropine eye drops (Fig. 1). Mean best-corrected visual acuity on presentation and at every follow-up was $0.1 \log$ MAR and near vision in all patients was N6 equivalent. Slit-lamp examination showed no evidence of any conjunctival congestion or corneal affliction at any visits. No systemic side effects were recorded.

Average photopic pupil size recorded on atropine therapy was $3.5 \mathrm{~mm}$ and a difference of $0.5 \mathrm{~mm}$ noted from baseline value.

Average NPA was $9 \mathrm{~cm}$ while on atropine $0.01 \%$. Average increase of NPA from baseline was $2.44 \pm 0.79 \mathrm{~cm}$ (Fig. 2).

Average increase in axial length was $0.12 \pm 0.11 \mathrm{D}$ over 6 months and $0.2 \pm$ $0.29 \mathrm{D}$ over 1 year ( $p$ value $=0.36$ ). Average increase in spherical equivalent over 6 months was $0.07 \pm 0.19 \mathrm{D}$ and over 1 year was $0.32 \pm 0.29 \mathrm{D}$ ( $p$ value $=0.32$ ).

There was no significant difference noted in the macular thickness after 1 year of atropine $0.01 \%$ usage ( $p$ value $=0.3$ ).

Five children had occasional glare to light and four children had occasional photophobia, not requiring any treatment for the same. One child had progression of $1.0 \mathrm{D}$ over 6 months period while on atropine $0.01 \%$ eye drops.

All cultures were negative. 
Table 1. Comparison of our study results with ATOM2 study using various concentrations of atropine

\begin{tabular}{|l|l|l|l|l|}
\hline Parameters & $\begin{array}{l}\text { Our study } \\
\text { (Atropine } \\
\mathbf{0 . 0 1 \% )}\end{array}$ & $\begin{array}{l}\text { ATOM2 } \\
\text { (Atropine } \\
\mathbf{0 . 0 1 \% )}\end{array}$ & Atropine 0.1\% & $\begin{array}{l}\text { Atropine } \\
\mathbf{0 . 5 \%}\end{array}$ \\
\hline $\begin{array}{l}\text { Allergic } \\
\text { conjunctivitis }\end{array}$ & Nil & Nil & Present in 4.1\% & $\begin{array}{l}\text { Present in } \\
4.1 \%\end{array}$ \\
\hline $\begin{array}{l}\text { Children using } \\
\text { progressive } \\
\text { photochromatic } \\
\text { glasses }\end{array}$ & Nil & $6 \%$ & All & All \\
\hline $\begin{array}{l}\text { Spherical } \\
\text { equivalent } \\
\text { increase over } \\
1 \text { year (D) }\end{array}$ & $0.32 \pm 0.29$ & $0.43 \pm 0.52$ & $0.31 \pm 0.50$ & $0.17 \pm 0.47$ \\
\hline $\begin{array}{l}\text { Axial length } \\
\text { increase over } \\
1 \text { year (mm) }\end{array}$ & $0.2 \pm 0.29$ & $0.24 \pm 0.19$ & $0.13 \pm 0.18$ & $0.11 \pm 0.17$ \\
\hline
\end{tabular}

\section{Discussion}

Atropine at $1.0 \%, 0.5 \%$, and $0.01 \%$ has been demonstrated through randomized trials to be effective in slowing myopia progression. ${ }^{12}$ Apart from effectiveness of medication, the long-term use of any medication calls for attention with regards to tolerability and compliance. However, the safety profile of atropine (systemic side effects and visual disturbances) often has been a source of concern and deterred many from using this medication.

Every unit increase in pupil size results in an exponential increase in the amount of light entering the eye, and this can cause glare and potential phototoxicity. Atropine, by its dilating and cycloplegic effect, might cause retinal phototoxicity and near vision problems necessitating the need for bifocal or progressive glasses for reading.

Atropine $0.01 \%$ has been proven to be effective in controlling progression of myopia. But atropine $0.01 \%$ was not commercially available when we started the study. If atropine needs to be used as a part of our clinical practice, it would need to be reconstituted. Contamination of the eye drops on storage becomes an additional concern apart from those listed above.

Our study collaborated findings of others ${ }^{13-15}$ in the absence of any systemic side effects with daily use over a period of 16 months. Atropine $0.01 \%$ was effective in reducing the progression of myopia by $91 \%$. There were no gross vision disturbances affecting their day-to-day life. All the children had near vision of N6 

myopic progression

equivalent. The retinal thickness remains unaffected by 1-year usage of the drug. There was no contamination of the eye drop at the end of 1 month.

The differences of our result from ATOM2 study may be due to the small sample size (Table 1).

There are some limitations in our study. First, this study is not a double-blind randomized design. Second, the small sample size and relatively short follow-up time are noted in our study. Long-term side effects may be apparent in a long period of follow up.

The lowest concentration of $0.01 \%$ atropine thus seems to retain efficacy in Indian children with darker iris and is a viable concentration for reducing myopia progression in children while being well-tolerated and safe from adverse effects. Even if this concentration is not commercially available in a locality, it can be safely reconstituted under laminar flow and used for controlling myopia progression in children.

\section{Conclusion}

Atropine $0.01 \%$ eye drops is safe and efficacious in controlling myopia progression in Indian children. Even if the commercially available preparation is not available, reconstituted atropine eye drops can be safely used.

\section{References}

1. World Health Organization. Elimination of Avoidable Visual Disability due to Refractive Errors (WHO/PBL/0079). Geneva, Switzerland: World Health Organization; 2000:Vision 2020.

2. Saw S-M. A synopsis of the prevalence rates and environmental risk factors for myopia. Clin Exp Optom. 2003;86:289-294.

3. Jain IS, Jain S, Mohan K. The epidemiology of high myopia—changing trends. Indian J Ophthalmol. 1983;31:723-728.

4. Mohan M, Pakrasi S, Zutshi R. Myopia in India. Acta Ophthalmol Suppl. 1988;185:19-23.

5. Goss DA, Hampton MJ, Wickham MG. Selected review on genetic factors in myopia. J Am Optom Assoc. 1988;59:875-884.

6. Mutti DO. Myopia - the nature versus nurture debate goes on. Invest Ophthalmol Vis Sci. 1996;37(6):952-957.

7. Yen MY, Liu JH, Kao SC, Shiao CH. Comparison of the effect of atropine and cyclopentolate on myopia. Ann Ophthalmol 1989;21:180-182, 187.

8. Shih YF, Chen $\mathrm{CH}$, Chou AC, et al. Effects of different concentrations of atropine on controlling myopia in myopic children. J Ocul Pharmacol Ther. 1999;15:85-90.

9. Shih YF, Hsiao CK, Chen CJ, et al. An intervention trial on efficacy of atropine and multi-focal glasses in controlling myopic progression. Acta Ophthalmol Scand. 2001;79:233-236.

10. Chua WH, Balakrishnan V, Chan YH, et al. Atropine for the treatment of childhood myopia. Ophthalmology. 2006;113:2285-2291.

11. Luu CD, Lau AM, Koh AH, Chua WH, Balakrishnan V, Tan D. Effects of long term atropine usage on retinal function. Invest Ophthalmol Vis Sci. 2003;44:4790.

12. Chia A, Chua W-H, Cheung Y-B, et al. Atropine for the treatment of childhood myopia: safety and efficacy of $0.5 \%, 0.1 \%$, and $0.01 \%$ doses (atropine for the treatment of myopia 2 ).

Ophthalmology. 2012;119(2):347-354. 
13. Wu PC, Yang YH, Fang PC. The long-term results of using low concentration atropine eye drops for controlling myopia progression in schoolchildren. J Ocul Pharmacol Ther. 2011;27(5):461-466.

14. Loughman J, Flitcroft DI. The acceptability and visual impact of $0.01 \%$ atropine in a Caucasian population. Br J Ophthalmol. 2016;100(11):1525-1529.

15. Nishiyama Y, Moriyama M, Fukamachi M, et al. Side effects of low dose atropine. Nippon Ganka Gakkai Zasshi. 2015;119(11):812-816. 